\title{
Le Centre neuromusculaire des Pays-Bas
}

\author{
Nicol Voermans, George Padberg, Marianne de Visser, Carlos Vrins, Leonard van den Berg
}

Les Pays-Bas

- 17 millions d'habitants

- 2 langues officielles : néerlandais et frison occidental (une langue co-officielle dans la province de Frise dans le nord)

- avec une densité de population de 414 personnes par $\mathrm{km}^{2}$ - 510 si l'on exclut l'eau - les

Pays-Bas sont classés comme un pays très densément peuplé

- 8 facultés de médecine, dont six hébergent un centre neuromusculaire reconnu (Figure 1);

- 800 neurologues dans 8 centres universitaires et 70 hôpitaux généraux

- l'assurance maladie standard est légalement obligatoire ; l'assurance complémentaire est facultative.

\section{Histoire}

Au milieu des années 1970, six centres neuromusculaires des centres médico-universitaires (CMU) des Pays-Bas ont créé une organisation virtuelle, le réseau de soutien interuniversitaire pour la recherche neuromusculaire, afin d'accroître et de partager dans le domaine neuromusculaire un niveau d'excellence en matière de soins, d'éducation et de recherche. Dans ce but, beaucoup de lobbying a été fait auprès des conseils d'administration desdits CMU pour la création de chaires professorales dans le domaine des maladies neuromusculaires. Le réseau a rapidement mis en place un congrès annuel pour partager les développements les plus marquants et offrir une plate-forme d'apprentissage pour tous les professionnels de santé impliqués dans le diagnostic et la prise en charge des patients neuromusculaires. Lors de réunions de consensus, l'expertise de chaque centre a été vite repérée et petit à petit, les neurologues des hôpitaux généraux ont orienté de plus en plus leurs patients vers ces centres spécialisés en pathologie neuromusculaire. Lorsque le Ministère de la Santé, du Bien-être et des Sports a demandé en 1986 aux CMU néerlandais de démontrer qu'il n'y avait pas trop de chevauchement entre les centres, notamment au niveau de la recherche, le réseau neuromusculaire a été cité comme un modèle exemplaire de collaborations fructueuses entre recherche médicale et recherche fondamentale.

\section{Centre neuromusculaire des Pays-Bas}

Le concept de réseau neuromusculaire, d'abord virtuel et informel, a évolué au fil des années pour devenir quelque chose de plus structuré : le Centre de ressources neuromusculaire des Pays-Bas (CNMPB) créé officiellement en 2017. Le CNMPB s'appuie sur les centres d'expertise neuromusculaire de six CMU néerlandais: Amsterdam, Leiden,
Maastricht, Nimègue, Rotterdam et Utrecht. Son objectif est d'améliorer la qualité de vie des patients atteints de maladies neuromusculaires par le biais d'une recherche scientifique de pointe, d'un excellent système d'éducation professionnelle de haut niveau, et d'une prise en charge optimisée et efficiente du patient neuromusculaire et d'une plateforme d'échanges partagée par les scientifiques et professionnels de santé. Les six centres couvrent les besoins diagnostiques et thérapeutiques de l'ensemble des maladies neuromusculaires acquises ou d'origine génétique. Par ailleurs, chaque centre dispose de son propre centre de recherche (Figure 1). Les patients sont en général bien disposés à se rendre dans d'autres centres neuromusculaires du pays pour participer à des projets de recherche et en particulier à des essais cliniques. Fidèle à l'ambition du CNMPB d'offrir des soins de meilleure qualité et mieux intégrés, des recommandations de bonnes pratiques en matière de diagnostic et de soins font l'objet de travaux de consensus entre les centres, et sont ensuite disponibles en ligne.

Avec près de 600 maladies neuromusculaires différentes, il est essentiel d'avoir une vision de l'ensemble de ce qui se fait en matière de recherche aux Pays-Bas et d'encourager les collaborations entre les groupes de recherche. Pour plusieurs maladies, les maladies du motoneurone, la dystrophie musculaire facio-scapulo-humérale (FSHD) et la dystrophie musculaire de Duchenne, des centres d'expertise interuniversitaires ont été ou sont en cours d'établissement afin de renforcer la recherche et la prise en charge des patients. Par exemple, le Centre des maladies du motoneurone tel que le Centre de la sclérose latérale amyotrophique (www.als-centrum.nl), est issu d'un partenariat très fructueux entre le Centre médical universitaire d'Utrecht et celui d'Amsterdam (Figure 1). 


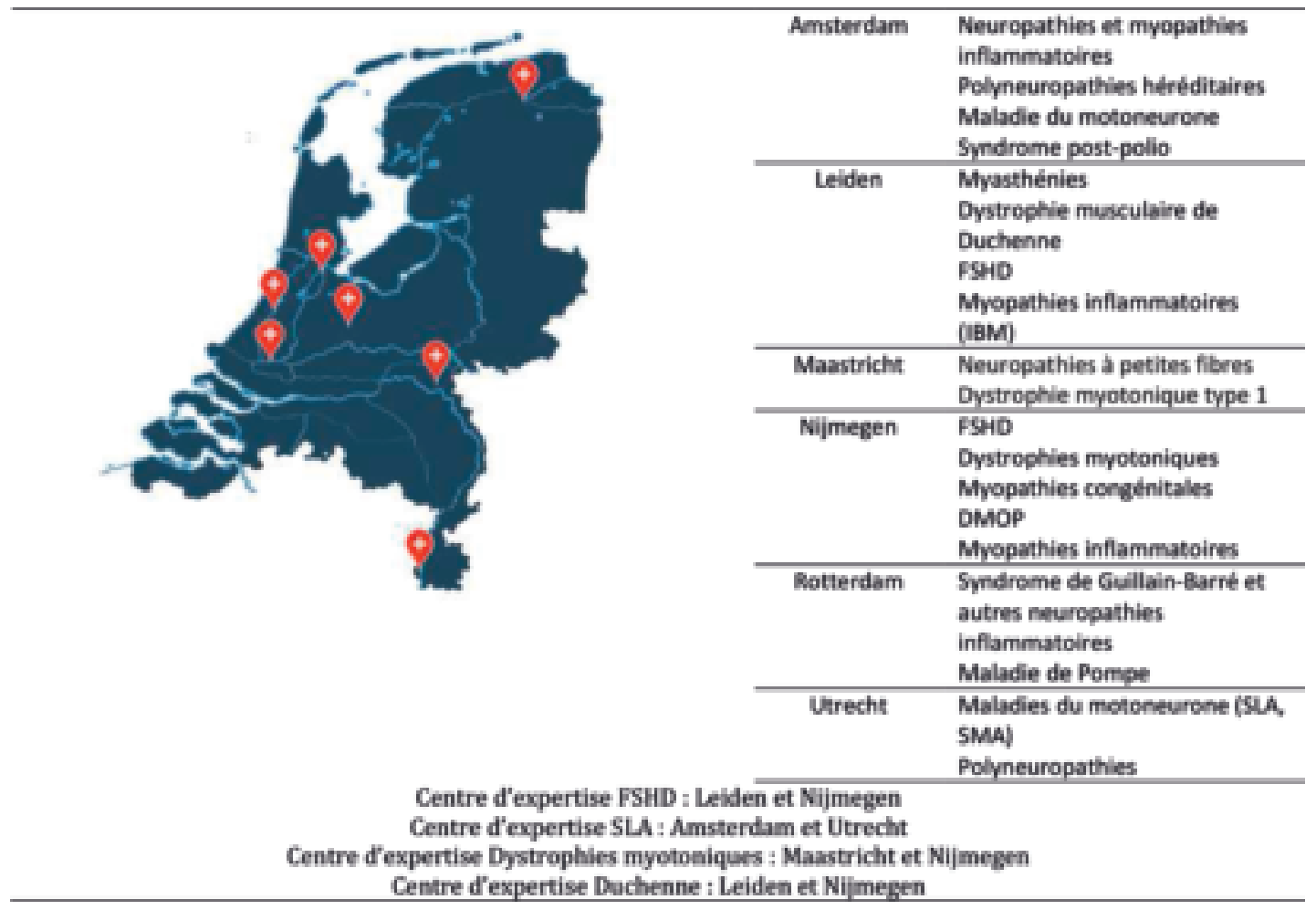

Figure 1

Le Centre Neuromusculaire des Pays-Bas.

Le partage des connaissances est essentiel dans l'esprit du CNMPB. En collaboration avec la Prinses Beatrix Muscle Foundation (voir ci-dessous), un symposium est organisé tous les ans pour les professionnels de santé et une réunion de deux jours intitulée Muscles2Meet est organisée tous les deux ans pour les chercheurs doctorants. Le CNMPB développe également un programme de détection et d'encouragement des jeunes talents parmi les chercheurs et médecins.

Les missions du Centre neuromusculaire des Pays-Bas

Sa mission est d'encourager la recherche neuromusculaire, de partager et de traduire de nouvelles connaissances au profit de la prise en charge et du traitement des enfants et des adultes atteints de maladies neuromusculaires.
2017 (https://ern-euro-nmd.eu). Les objectifs du réseau sont de : faciliter l'accès au diagnostic et aux traitements, fournir à tous les citoyens européens des soins de santé abordables, efficients et de haut niveau, promouvoir les collaborations en partageant l'expertise, faciliter le partage des connaissances et en maximisant les synergies entre les États membres, et en promouvoir l'égalité en regard des soins, quel que soit le lieu de résidence du patient en Europe.

Avec trois membres néerlandais au sein du comité exécutif de l'ERN Euro-NMD et le concours du CNMPB dans son ensemble, les Pays-Bas prennent une large part à cette nouvelle initiative visant à harmoniser et à améliorer les soins de santé et la recherche neuromusculaires à travers l'Europe.

Le CNMPB organise régulièrement des réunions avec des collègues des pays voisins. Il existe une longue tradition de réunions de recherche semestrielles avec la Belgique. Le club d'étude neuromusculaire belgo-néerlandais a célébré sa $100^{e}$ réunion en 2016. Tous les trois ans, est organisée une réunion conjointe avec les collègues allemands et belges à Vaals, située à la frontière des trois pays. Il en est de même avec les liens établis avec la British Myology Society. 
Le triangle d'or : collaboration

avec les organisations caritatives

\section{et associations de patients}

En 1956, une Fondation pour la prévention contre la poliomyélite (Fonds ter bestrijding van Kinderverlamming) a été créée sous le haut patronage de la princesse Beatrix d'Oranje. La Fondation porte son nom dès 1957 et se consacre principalement aux maladies neuromusculaires, une fois disparue la poliomyélite suite à l'introduction de la vaccination. Toujours Présidente de la Fondation - connue sous le nom Prinses Beatrix Muscle Foundation - la princesse est encore actuellement activement impliquée dans certaines de ses activités. L'objectif principal de la Fondation est d'offrir une aide financière à la recherche sur le traitement curatif des maladies neuromusculaires (MNM) et d'améliorer la qualité de vie des patients atteints de MNM. Les chercheurs et médecins impliqués dans les MNM sont encouragés à postuler pour des subventions et des bourses de recherche dans le domaine neuromusculaire. La Fondation fournit également une plateforme réservée aux jeunes chercheurs en MNM pour partager leurs connaissances.

Au fil des ans, quatre organisations caritatives spécialisées dans les MNM ont vu le jour, visant à stimuler et soutenir la recherche, notamment la FSHD
Foundation, the Duchenne Parents' Project, la Fondation SLA et "Spieren voor Spieren ". Ces quatre fondations et la Fondation Prinses Beatrix collaborent avec l'association des patients neuromusculaires ('Spierziekten Nederland') pour organiser conjointement un grand symposium annuel. Lors des nombreuses sessions parallèles, médecins et chercheurs informent les patients sur les dernières avancées de la recherche et découvrent en retour les besoins spécifiques exprimés par les patients.

Le CNMPB entretient une collaboration intense et fructueuse avec Spierziekten Nederland et forme, avec les cinq fondations de recherche, un véritable " triangle d'or ". Les fondations de recherche apportent un soutien financier à un grand nombre de projets de recherche au sein du CNMPB et permettent également de sensibiliser la population néerlandaise aux MNM. Les membres de Spierziekten Nederland participent à la conception des projets de recherche et à l'interprétation des résultats. L'association de patients est également active dans l'amélioration des aspects généraux de la prise en charge neuromusculaire, comme en cas d'urgence médicale, et fournit des informations actualisées sur toutes les (catégories de) MNM pour lesquelles ils consultent leurs conseillers médicaux, spécialistes experts du CNMPB.

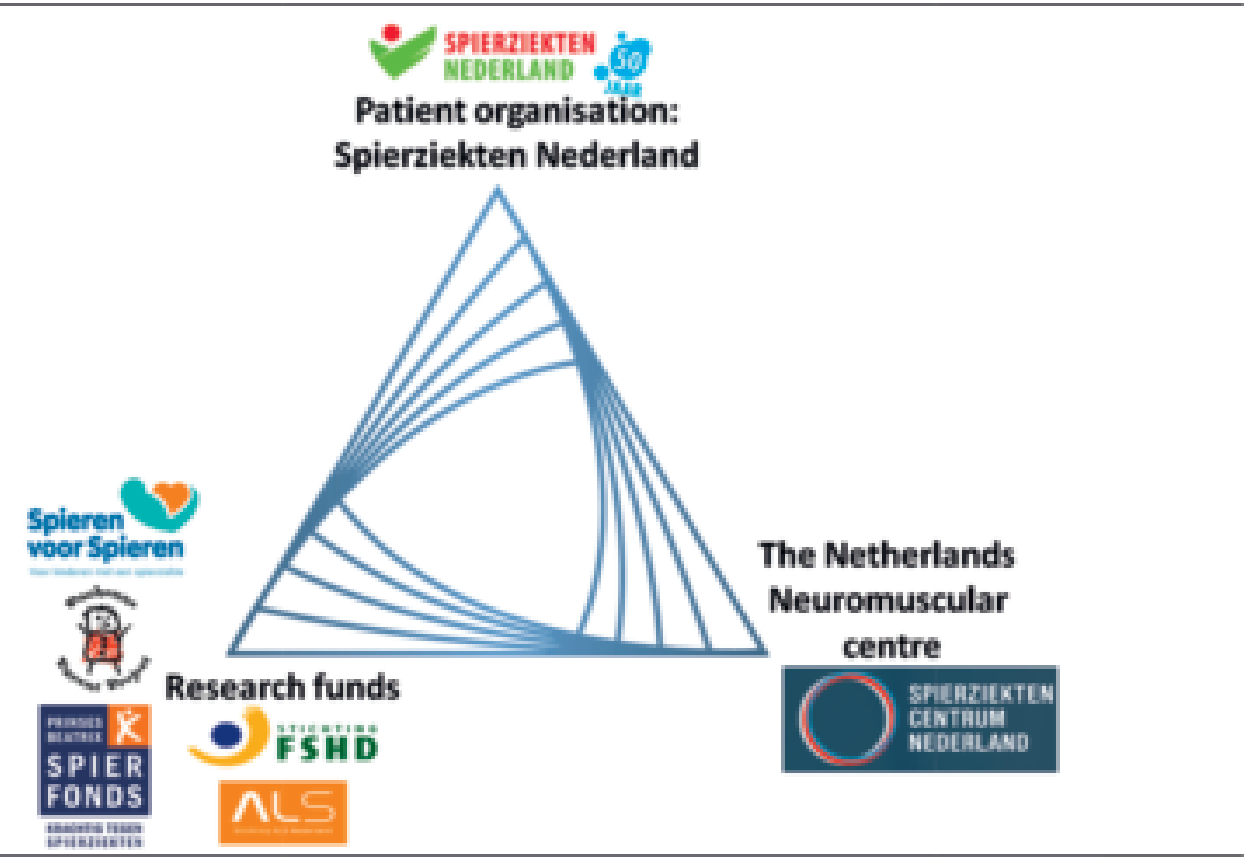

Figure 2

La collaboration entre le CNMPB, l'organisation des patients et les Fondations de recherche. 


\section{Les maladies neuromusculaires aux Pays-Bas}

Depuis sa création en 2004, la base de données nationale néerlandaise CRAMP (pour Computer Registry of All Myopathies and Polyneuropathies ou Registre informatique de toutes les myopathies et polyneuropathies) recense les personnes souffrant de pathologie neuromusculaire [1]. CRAMP était le premier registre national qui ne se concentrait pas sur un unique groupe de maladies. Il a ainsi permis l'enregistrement d'un grand nombre de patients neuromusculaires, y compris ceux sans diagnostic précis mais présentant des signes d'appel neuromusculaires (myalgies, crampes, amyotrophie, autres). L'une des motivations à remplir le registre résidait dans la perspective d'avoir des données épidémiologiques nationales à jour sur les MNM pour les Pays-Bas. Ceci a permis d'aboutir à une estimation de la prévalence et à des chiffres sur le genre et la date d'apparition des symptômes pour 30 maladies neuromusculaires [2]. Le NMCN développe actuellement un système de base de données plus sophistiqué s'appuyant sur la base de données CRAMP. La nouvelle configuration permettra de relier la base en toute sécurité au système informatique de chaque hôpital et réduira de manière significative les démarches administratives qui devaient auparavant être exécutées par le médecin traitant. CRAMP 2.0 sera lancé en 2018 et se traduira par une collecte des données plus précise et un taux d'exhaustivité accru. La nouvelle base de données fournira un aperçu fiable de la distribution des MNM et constituera non seulement un excellent point de départ pour des études de recherche épidémiologique et clinique, mais contribuera aussi de manière significative à la préparation de futurs essais cliniques aux Pays-Bas.

\section{The Neuromuscular Center of the Netherlands}

\section{LIENS D'INTÉRÊTT}

Les auteurs déclarent n'avoir aucun lien d'intérêt concernant les données publiées dans cet article.

\section{RÉFÉRENCES}

1. Van Engelen BG, van Veenendaal H, van Doorn PA, et al. The Dutch neuromuscular database CRAMP (computer registry of all myopathies and polyneuropathies): development and preliminary data. Neuromuscul Disord 2007 ; 17 : 33-7.

2. Deenen JC, van Doorn PA, Faber CG, et al. The epidemiology of neuromuscular disorders: Age at onset and gender in the Netherlands. Neuromuscul Disord 2016 ; 26 : 447-52.

\section{LEXIQUE}

$\mathrm{CMU}$ : centre médico-universitaire

CNMPB : centre de ressources neuromusculaire des Pays-Bas

CRAMP (Computer Registry of All Myopathies and Polyneuropathies) : Registre informatique de toutes les myopathies et polyneuropathies

ERN-Euro-NMD : Réseau européen de référence pour les maladies neuromusculaires rares

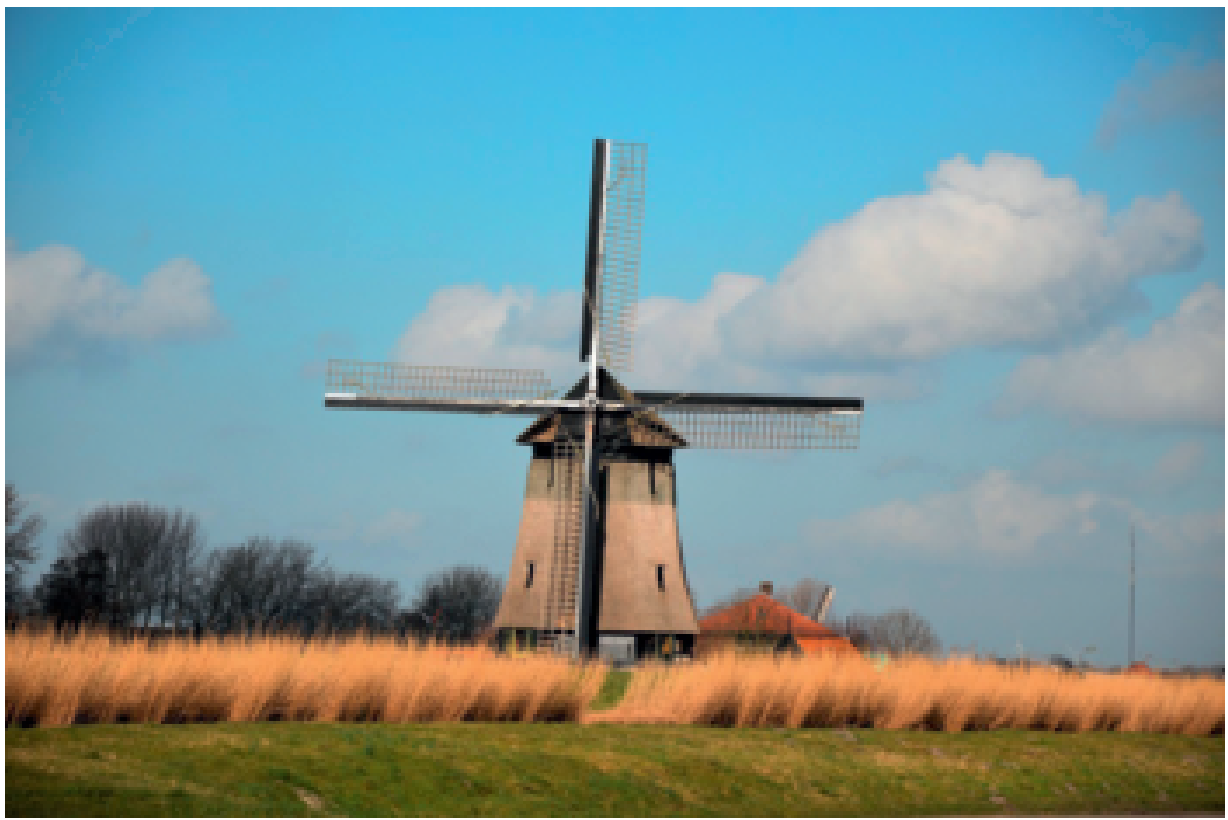

https://pixabay.com/fr/moulin-\%C3\%A0-vent-pays-bas-tradition-316029/(CC0 Creative Commons). 